\title{
Reduced Activity of von Willebrand Factor after Flow- Diverting Stent Implantation for Intracranial Aneurysms: A Link to Acquired von Willebrand Disease?
}

\author{
(DI. Oran, (DC. Cinar, (D). Bozkaya, (D). Parildar, and (D). Duman
}

\begin{abstract}
BACKGROUND AND PURPOSE: Vascular devices generating high shear stress can cause type 2A acquired von Willebrand disease, which is characterized by low von Willebrand factor activity accompanied by hemorrhagic complications. The braided mesh structure of flow-diverting stents with a relatively small strut size can create abnormally high shear stress while arterial blood flows through the stent struts into the aneurysm, and flow-diverting stent may be associated with reduced von Willebrand factor activity.
\end{abstract}

MATERIALS AND METHODS: Aneurysmal morphologic parameters and patient data were examined retrospectively among patients who had an unruptured intracranial aneurysm treated with a flow-diverting stent. The RISTOtest (test for whole blood ristocetininduced platelet aggregation) for von Willebrand factor activity, as well as tests for aspirin and clopidogrel/prasugrel effectiveness, were performed immediately before the endovascular procedure and 24 hours later by multiple electrode aggregometry.

RESULTS: A total of 39 patients with 56 aneurysms were recruited, and statistical analyses were performed in 32 patents with 49 aneurysms. Compared with the baseline values, von Willebrand factor activity was reduced in 16 patients but increased in 23 patients. Aneurysmal variables (eg, neck area, volume, volume-to-neck area ratio, size ratio, and morphologic index) clearly distinguished patients with reduced von Willebrand factor activity from those with nonreduced von Willebrand factor activity. The receiver operating characteristic curve showed that the morphologic index and volume had the highest discriminative power, with an area under the curve of 0.99 .

CONCLUSIONS: In high-volume/large-neck aneurysms, flow-diverting stent implantation can cause reduced von Willebrand factor activity, which may be linked causally to acquired von Willebrand disease.

ABBREVIATIONS: ADP = test for clopidogrel/prasugrel responsiveness; AvWD = acquired von Willebrand disease; FDS = flow-diverting stent; LVAD = left ventricular assist device; $\mathrm{VWD}=$ von Willebrand disease; $\mathrm{vWF}=$ von Willebrand factor; RISTO $=$ test for whole blood ristocetin-induced platelet aggregation

$\mathbf{R}$ educed von Willebrand Factor (vWF) activity, secondary to quantitative and qualitative abnormalities, results in defective hemostasis known as von Willebrand disease (vWD), the most frequent hereditary hemorrhagic disease worldwide. Various cardiovascular diseases, such as aortic stenosis and several congenital heart diseases, have long been known to cause acquired von Willebrand disease (AvWD). ${ }^{1}$ In recent decades, a considerable number of patients have also been described with AvWD, associated with vascular devices that create pathologically high shear stress in the blood. Such devices include the left ventricular assist

Received August 9, 2019; accepted after revision October 20

From the Departments of Radiology (I.O., C.C., H.B., M.P.) and Internal Medicine (S.D.), Ege University Medical School, Izmir, Turkey. Dr Oran is currently affiliated with Section of Interventional Radiology, KENT Hospital, Izmir, Turkey.

Please address correspondence to Ismail Oran, MD, EBIR, Section of Interventional Radiology, KENT Hospital, 35630, Cigli, Izmir, Turkey;

e-mail: ismailoran@gmail.com; @profismailoran

http://dx.doi.org/10.3174/ajnr.A6343 device (LVAD) and extracorporeal membrane oxygenation. ${ }^{2,3}$ vWF is synthesized normally and at normal levels in such patients, and the reduced vWF activity results from accelerated enzymatic cleavage of the factor due to device-related high shear stress.

The flow-diverting stent (FDS) is a novel endovascular device for the treatment of previously difficult-to-treat cerebral aneurysms. A unique feature of the FDS is its braided mesh structure with a relatively high pore density and small strut size. Computational fluid dynamics simulation recently showed that FDSs ${ }^{4}$ and even peripheral stents ${ }^{5}$ can create abnormally high supraphysiologic shear stress while blood flow passes through stent struts into the aneurysm.

We aimed to characterize reduced vWF activity after FDS implantation for endovascular treatment of intracranial aneurysms. We focused on the spatial characteristics of the aneurysm to identify $\geq 1$ simple variable that may predict the presence of reduced vWF activity. We also assessed the current literature about the vascular device-related AvWD in the 
context of our results to propose a hypothesis that links reduced vWF activity to delayed intracranial bleeding after FDS implantation, a life-threatening complication that occurs in approximately $6 \%$ of these patients. ${ }^{6}$

\section{MATERIALS AND METHODS Patients}

This study was performed in accordance with the ethical standards established in the 1964 Declaration of Helsinki and its later amendments, and the study protocol was approved by our institutional review board. All patients provided written informed consent to undergo planned endovascular procedures. Data were collected retrospectively from patients who had intracranial aneurysms treated endovascularly with an FDS (Pipeline Embolization Device; Covidien, Irvine, California) (alone or in conjunction with coiling) from July 2017 to October 2018. Patients received FDS treatment if they had giant, wide-neck, fusiform, dissecting, and/or recurrent/remnant (after intrasaccular coiling) unruptured aneurysms or any unruptured aneurysms deemed unsuitable for standard (simple coiling, balloon-assisted coiling, or stent-assisted coiling) endovascular procedures. Patients with multiple aneurysms were included only if all aneurysms had received FDS treatment. Only patients treated by dual-antiaggregant loading with a single dose (at midnight the night before a morning endovascular procedure) were selected; all other loading strategies were excluded. Finally, patients who had an existing mass effect and were given intravenous and/or oral corticosteroids during/immediately after FDS implantation were also excluded from the study, due to possible distortion of the aggregometry results.

\section{Aneurysm Characteristics}

All geometric characteristics were examined on reconstructed $3 \mathrm{D}$ rotational angiographic images (Allura Clarity; Philips Healthcare, Best, the Netherlands) by 2 interventional neuroradiologists. The measured morphologic parameters of the aneurysms included neck width (along the long axis of the parent artery), height (perpendicular to the neck), and 2 orthogonal maximum widths, represented as $\mathrm{N}, \mathrm{H}, \mathrm{W} 1$, and $\mathrm{W} 2$, respectively. The diameter of the parent artery (D) was also measured at the level of the neck region. All measurements were expressed in millimeters. In patients with bifurcation aneurysms, the arithmetic mean diameters of the main trunk and 1 stented branch were calculated. In patients with fusiform aneurysms, the neck width was measured as the aneurysm length along the parent artery. Using these parameters, we calculated specific variables previously defined in the literature, ${ }^{7}$ including the aspect ratio (calculated as $\mathrm{H} / \mathrm{N}$ ), size ratio (H/D), neck area, volume of the aneurysm, and volume-to-neck area ratio. To generate rapid and user-friendly calculations, we assumed that the neck area was circular and calculated it simply as $\mathrm{N}^{2}$; volume was calculated as $\mathrm{H} \times$ $\mathrm{W} 1 \times \mathrm{W} 2$ (for saccular aneurysms). We defined 1 novel variable (morphologic index), multiplication of the neck area and volume, represented as $\mathrm{N}^{2} \times \mathrm{H} \times \mathrm{W} 1 \times \mathrm{W} 2$. In patients with multiple aneurysms, individual variables were calculated for each aneurysm and were then added to obtain a single value for each patient.

\section{Medications}

All endovascular procedures were performed with the patients under general anesthesia and systemic heparinization, which was typically initiated before placement of the guiding catheter to maintain the activated clotting time at 2- to 3-fold above baseline (initial heparin bolus, 5000-10,000 IU). All patients received a loading dose of $600 \mathrm{mg}$ of clopidogrel or 30-60 mg of prasugrel, as well as $300 \mathrm{mg}$ of aspirin, 9-12 hours before the procedure. As a maintenance dose, $75 \mathrm{mg}$ /day of clopidogrel or $10 \mathrm{mg} /$ day of prasugrel, combined with 100-300 mg of aspirin, was prescribed to all patients at the time of discharge. Some patients with a relatively low body mass index could not tolerate the prasugrel-aspirin dual-maintenance therapy; aspirin was discontinued in such circumstances. In patients undergoing stent implantation in the carotid artery proximal to the Willis polygon or vertebral artery, clopidogrel/prasugrel maintenance therapy was typically given for up to 3 months. In patients who underwent stent implantation in or distal to the Willis polygon or basilar artery, antiaggregant maintenance was extended by up to 6 months. After cessation of clopidogrel or prasugrel, aspirin was prescribed for at least another 6 months.

\section{Impedance Aggregometry}

Patients were assessed for platelet responsiveness by a rapid platelet function test (Multiplate Analyzer; Roche, Basel, Switzerland) performed in the angiography suite, just before the endovascular procedure. During the past 2 years, we have routinely repeated the test at 24 hours after treatment for patients treated with FDSs. We also expanded our routine test examination to include the ristocetin-induced platelet aggregation (RISTOHigh; Roche) (test for vWF activity) tests, besides the test for clopidogrel/prasugrel responsiveness (ADP), the test for aspirin responsiveness, and the test for general performance of platelets and their glycoprotein IIb/ IIIa receptor. All 4 tests were performed through a single blood draw and lasted about 10 minutes. As described previously, we followed a tailored medication strategy and noted a positive clinical impact under the guidance of the rapid platelet-function test. ${ }^{8}$

Patients were scheduled for follow-up visits at 1, 3, and 6 months after discharge from our institution. Routine CTA was performed at 3 months, and CTA or digital subtraction angiography was performed at 6 or 12 months after discharge.

\section{Statistical Analyses}

Data were analyzed using SPSS (Version 25; IBM, Armonk, New York). Differences with $P<.05$ were considered statistically significant. Categoric data were expressed as No. (\%) and were compared using the Fisher exact test. Continuous data were expressed as the mean $\pm \mathrm{SD}$ and were compared using the Mann-Whitney $U$ test. The Wilcoxon signed rank test was used to compare dependent data. Receiver operating characteristic curve analysis was performed to determine the optimal sensitivity and specificity, as well as the cutoff points to establish the predictive ability of each variable.

\section{RESULTS}

A total of 39 patients with 56 aneurysms were recruited (patient age, 18-67 years). Of the 56 aneurysms, 42 were sidewall and 14 

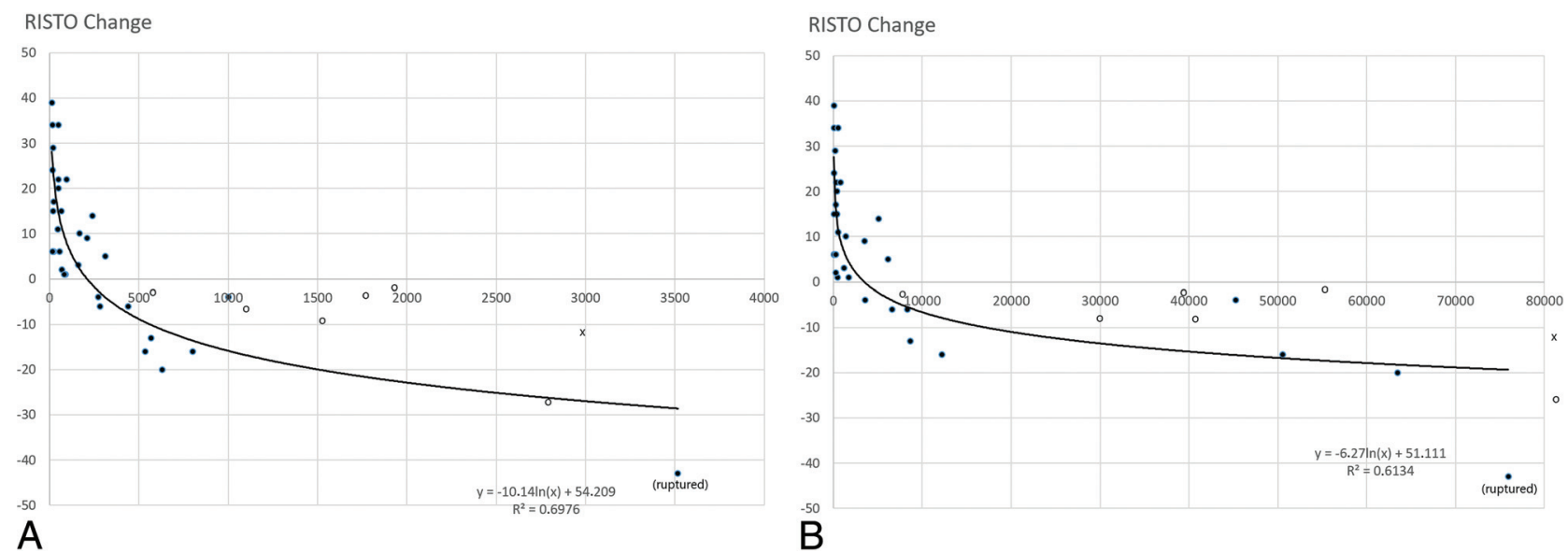

B

FIG 1. Scatterplots extracted from 32 patients treated by FDS only (closed circles) show a comparison of RISTO (ie, whole blood ristocetininduced platelet aggregation) change with the volume $(A)$ and morphologic index $(B)$ of the aneurysm depicted on the $x$-axis. Open circles denote 6 patients treated by coiling + FDS, and $x$ denotes 1 fusiform aneurysm; all have actually reduced vWF activity. The patient with delayed aneurysmal bleeding after FDS implantation is designated by (ruptured).

Table 1: Treatment variables in the reduced and nonreduced vWF groups

\begin{tabular}{lcccc}
\hline \multicolumn{1}{c}{ Characteristic } & $\begin{array}{c}\text { Reduced } \\
\text { vWF (\%) }\end{array}$ & $\begin{array}{c}\text { Nonreduced } \\
\text { vWF (\%) }\end{array}$ & All (\%) & $\boldsymbol{P}$ Value \\
\hline Patient No. & 9 & 23 & 32 & \\
Mean age (yr) & $59.4 \pm 5.6$ & $46.8 \pm 11$ & $50.3 \pm 11.3$ & .003 \\
Sex (female) & $7(77.7)$ & $17(73.9)$ & $24(75)$ & .869 \\
Mean aneurysm No. & $1.56 \pm 0.7$ & $1.61 \pm 0.5$ & $1.59 \pm 0.6$ & .742 \\
Aneurysm frequency & & & & \\
$\quad 1$ aneurysm/patient & $5(55.6)$ & $13(56.5)$ & $18(56.3)$ & .559 \\
2 aneurysms/patient & $3(33.3)$ & $9(39.1)$ & $12(37.5)$ & .564 \\
$\quad 3$ aneurysms/patient & $1(11.1)$ & $1(4.4)$ & $2(6.2)$ & .826 \\
FD frequency & & & & \\
$\quad 1$ stent/patient & $5(55.6)$ & $17(73.9)$ & $22(68.7)$ & .566 \\
2 stents/patient & $3(33.3)$ & $5(21.7)$ & $8(25)$ & .581 \\
3 stents/patient & $1(11.1)$ & $1(4.4)$ & $2(6.3)$ & .294 \\
\hline
\end{tabular}

included in the reduced-vWF group. The remaining 23 patients did not show a reduction in vWF activity and thus composed the nonreduced vWF group. The demographic data and treatment variables in the reduced and nonreduced vWF groups are summarized in Table 1. All test results and aneurysmal morphologic variables of the reduced and nonreduced vWF groups are summarized in Tables 24. After we compared all aneurysmal variables with the RISTOtest results, the so-called morphologic index and volume of the aneurysm provided superior were bifurcation aneurysms; 1 aneurysm was fusiform and the remaining 55 were saccular aneurysms. Five and 37 were located on the cavernous and intradural segments of the internal carotid artery, respectively; 10, on the middle cerebral artery; 1 , on the anterior cerebral artery; 2 , on the vertebral artery; and 1, on the basilar artery. All patients were treated with FDSs; 6 patients ( 1 aneurysm each) also received suboptimal coiling before FDS implantation during the same procedure. This preceding coiling was performed for the treatment of aneurysms with relatively large sizes that exceeded 1.5$2 \mathrm{~cm}$ in 1 dimension.

The RISTOtest results indicated reduced vWF activity 24 hours after treatment, compared with baseline values, in 16 (41\%) patients. The preceding preventive intrasaccular coiling may further change flow variables within the aneurysmal lumen and distort the RISTOtest results; thus, the 6 patients treated by coiling plus an FDS were not included in the statistical analyses. Indeed, all those 6 patients had reduced vWF activity (Fig1). One patient with fusiform aneurysms and reduced vWF activity was also excluded from the analysis. After we excluded these 7 patients, 9 of the remaining patients with 14 saccular aneurysms treated by an FDS alone were discrimination between the reduced and nonreduced vWF groups (Table 5 and Figs 1 and 2).

All patients were followed up both radiologically and clinically for at least 3 months. We encountered 2 clinical complications. First, 1 patient from the reduced vWF group with 2 aneurysms at the basilar artery fenestration and right MCA bifurcation experienced dysphasia 10 days after treatment. MR imaging showed right-sided insular cortex ischemia. At 3 months, the dysphasia had resolved completely, and both aneurysms were occluded according to CTA. Second, 1 patient from the reduced vWF group with 3 aneurysms at the anterior communicating artery and right and left MCA bifurcation experienced rupture of a large (18-mm maximum diameter) anterior communicating artery aneurysm into the ventricular system 4 weeks after treatment, which subsequently resulted in death. For anatomic reasons, preventive coiling before FDS implantation was not used for this relatively large aneurysm. No other clinical complications were observed during the 3-month follow-up period.

\section{DISCUSSION}

This study is the first to show laboratory evidence of reduced vWF activity in approximately $41 \%$ of our study population after 
FDS implantation for the treatment of intracranial aneurysms. Reduced vWF activity was best predicted by the morphologic index and volume of the aneurysm, with cutoff values of 4343 and 256, respectively. This finding suggests that a FDS may reduce vWF activity after its implantation in a saccular aneurysm larger than $8-10 \mathrm{~mm}$ in 1 dimension, according to the results of Multiplate Analyzer. Our findings clearly link the FDS

Table 2: Multiplate aggregometry results in all 32 patients

\begin{tabular}{lcccc}
\hline Aggregometry & $\begin{array}{c}\text { Initial } \\
\text { (Mean) }\end{array}$ & Last (Mean) & $\boldsymbol{P}$ Value & $\begin{array}{c}\text { Normal } \\
\text { Range }^{\mathbf{a}}\end{array}$ \\
\hline ADP & $13.34 \pm 5.4$ & $11.97 \pm 5.2$ & .096 & $53-122$ \\
ASPI & $12.0 \pm 5.0$ & $12.78 \pm 6.4$ & .537 & $74-136$ \\
TRAP & $57.34 \pm 23.6$ & $60.0 \pm 26.6$ & .524 & $94-156$ \\
RISTOtest & $31.88 \pm 17.9$ & $38.66 \pm 20.3$ & .024 & $90-201$ \\
\hline
\end{tabular}

Note:-ASPI indicates test for aspirin responsiveness; TRAP, test for general performance of platelet and its glycoprotein IIb/IIla receptor; RISTO, test for whole blood ristocetin-induced platelet aggregation.

${ }^{a}$ According to the manufacturer.

Table 3: Multiplate aggregometry results among the reduced and nonreduced vWF groups

\begin{tabular}{lccc}
\hline $\begin{array}{c}\text { Multiplate } \\
\text { Aggregometry }\end{array}$ & $\begin{array}{c}\text { Reduced vWF } \\
\text { (Mean) }(\boldsymbol{n}=\mathbf{9})\end{array}$ & $\begin{array}{c}\text { Nonreduced vWF } \\
\text { (Mean) }(\boldsymbol{n}=\mathbf{2 3})\end{array}$ & $\begin{array}{c}\boldsymbol{P} \\
\text { Value }\end{array}$ \\
\hline ADPin & $14.78 \pm 5.5$ & $12.78 \pm 5.4$ & .433 \\
ADPlast & $12.33 \pm 6$ & $11.83 \pm 5.1$ & .681 \\
ASPlin & $11.67 \pm 5.6$ & $12.13 \pm 4.9$ & 1.000 \\
ASPIlast & $14.56 \pm 6.5$ & $12.09 \pm 6.4$ & .341 \\
TRAPin & $59.11 \pm 25.3$ & $56.65 \pm 23.5$ & .837 \\
TRAPlast & $50.44 \pm 16.2$ & $63.74 \pm 29.2$ & .229 \\
RISTOin & $43.44 \pm 21.9$ & $27.35 \pm 14.3$ & .103 \\
RISTOlast & $29.22 \pm 18.1$ & $42.35 \pm 20.3$ & .094 \\
RISTOchange & $-(14.22 \pm 12.3)$ & $+(15.0 \pm 11.3)$ & .000 \\
RISTOchange & -43 to -4 & +1 to +39 & \\
$\quad$ (range) & & & \\
\hline
\end{tabular}

Note:--in indicates initial; -last, last; ASPI, test for aspirin responsiveness; TRAP, test for general performance of platelet and its glycoprotein IIb/IIla receptor; RISTO, test for whole blood ristocetin-induced platelet aggregation.

Table 4: Morphologic variables of aneurysms in the reduced and nonreduced vWF groups

\begin{tabular}{lccc}
\hline $\begin{array}{c}\text { Morphologic } \\
\text { Variables }\end{array}$ & $\begin{array}{c}\text { Reduced vWF } \\
\text { (Mean) }(\boldsymbol{n}=\mathbf{9})\end{array}$ & $\begin{array}{c}\text { Nonreduced } \\
\text { vWF (Mean) } \\
(\boldsymbol{n}=\mathbf{2 3})\end{array}$ & $\begin{array}{c}\boldsymbol{P} \\
\text { Value }\end{array}$ \\
\hline Neck area & $41.35 \pm 32.89$ & $11.44 \pm 6.13$ & .000 \\
Volume & $894.09 \pm 1010.45$ & $82.33 \pm 81.37$ & .000 \\
$\begin{array}{l}\text { Volume-to- } \\
\quad \text { neck area }\end{array}$ & $37.11 \pm 49.4$ & $9.65 \pm 7.12$ & .001 \\
$\begin{array}{l}\text { Aspect ratio } \\
\text { Size ratio }\end{array}$ & $1.72 \pm 0.74$ & $1.31 \pm 0.43$ & .103 \\
$\begin{array}{l}\text { Morphologic } \\
\text { index }\end{array}$ & $30,515.58 \pm 28,234.38$ & $1042.84 \pm 1641.06$ & .000 \\
\hline
\end{tabular}

implantation to AvWD and thus may provide a way to better explain the mechanism of delayed hemorrhagic complications because both the laboratory-verified reduced vWF activity and various hemorrhagic complications during the clinical course are 2 leading characteristics of shear-generating vascular devicerelated type $2 \mathrm{~A}$ AvWD. ${ }^{1-3}$

vWF mediates platelet tethering to the subendothelial matrix at sites of vascular injury to initiate hemostasis under the condition of arterial flow. Its secretion from endothelial cells is constitutive and accelerated in response to inflammation and ischemia (ie, activated endothelium). A mature vWF monomer has several domains and forms high-molecular-weight multimers (up to 100 monomers) just before entering the circulation; the A1 domain contains binding sites for the platelet glycoprotein Ib-IX receptor complex, whereas the A3 domain binds to subendothelial collagen. The A2 domain has a specific site that is cleaved by disintegrin and metalloprotease with thrombospondin type 1 repeat 13 (ADAMSTS-13) normally found in the circulation. The vWF multimer circulates normally in the globular inactive form and is hemostatically active only after exposure to supraphysiologic high shear stress, which causes force-induced conformational changes (ie, elongation), which lead to brief activation of the binding domains of A1 and A3. The vWF attains a globular configuration within 1-2 seconds when the shear is stopped or normalized. This shear-induced elongation, particularly in a repetitive manner, also renders the vWF multimer accessible to the ADAMTS-13 enzyme, leading to smaller vWF multimer fragments, which globally reduce factor activity. ${ }^{9}$

Although many studies have used computational fluid dynamics to analyze changes in intrasaccular flow and shear stress on the aneurysm wall after FDS implantation, 1 study also examined the shear stress of blood flow through stent struts in the aneurysm. ${ }^{4}$ The authors of that study found ultra-high levels of blood shear stress (up to $200 \mathrm{~Pa}$ ) through the struts, nearly equal to the shear stress created by a typical LVAD. The authors stated a possible link existed between shear stress-induced platelet activation and subsequent intrasaccular white thrombus formation after FDS. That study, however, did not refer to vWF or AvWD. Another computational fluid dynamics study, in which a bare metal stent was implanted across an aneurysm of the thoracic aorta, also yielded similar results, with a peak value of the systolic shear rate through the stent struts of up to 16,000 /s (approximately 40 - to $50-\mathrm{Pa}$ shear stress). ${ }^{5}$ Of note, a critical shear rate of $3000-5000 / \mathrm{s}$ (approximately 5- to 8-Pa shear stress) is needed for vWF elongation (ie, activation). ${ }^{10}$

We have used the Multiplate Analyzer, a rapid automated whole-blood aggregometry test, for determination of vWF activity because all patients have already been assessed by this test for

Table 5: Cutoff values of aneurysm variables according to the ROC analysis

\begin{tabular}{lcccccc}
\hline Morphologic Variables & AUC & Threshold Value & Sensitivity (\%) & Specificity (\%) & 95\% Confidence Intervals & $\boldsymbol{P}$ Value \\
\hline Neck area & 0.908 & 18.35 & 78 & 79 & $0.800-1.000$ \\
Volume & 0.990 & 256 & 89 & 96 & $0.966-1.000$ & .000 \\
Volume-to-neck area & 0.870 & 13.67 & 89 & 78 & $0.736-1.000$ \\
Aspect ratio & 0.691 & 1.47 & 67 & 65 & $0.486-0.895$ \\
Size ratio & 0.908 & 2.16 & 89 & 78 & $0.807-1.000$ \\
Morphologic index & 0.990 & 4343 & 89 & 91 & $0.965-1.000$ \\
\hline
\end{tabular}

Note:-AUC indicates area under curve; ROC, receiver operating characteristic. 


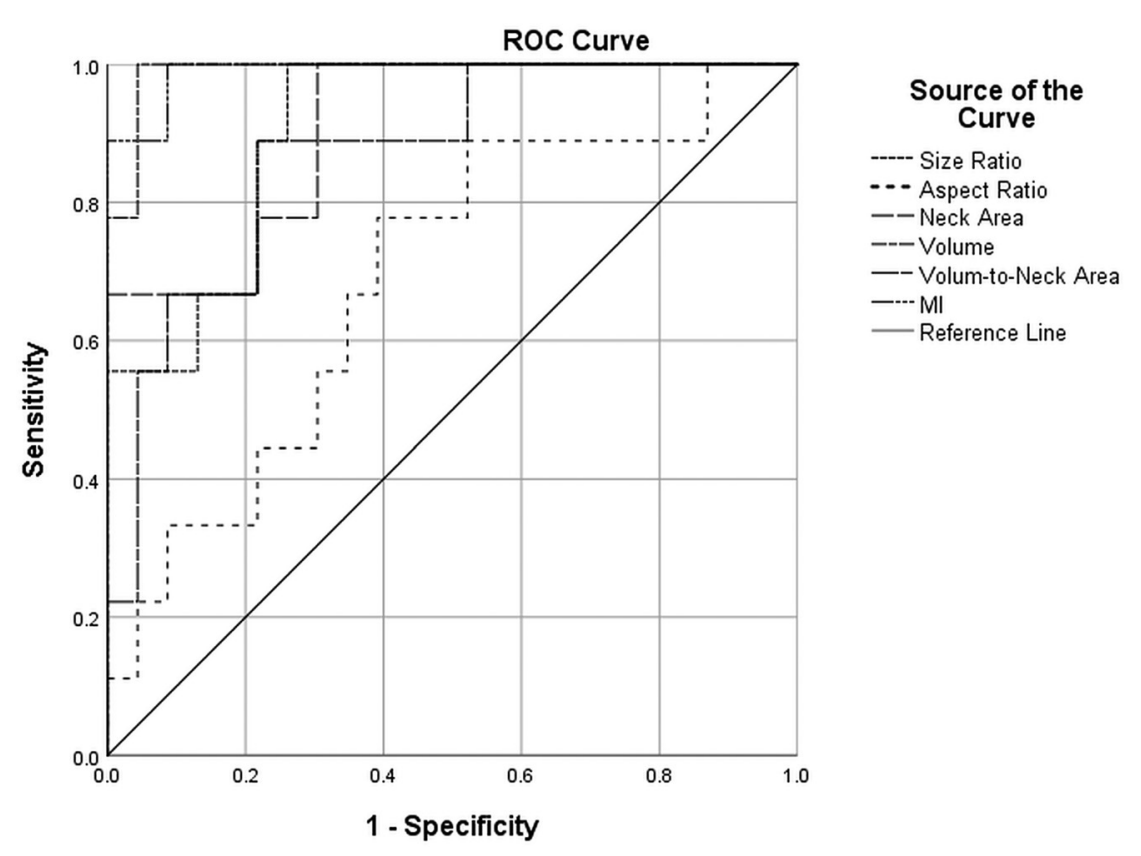

FIG 2. Receiver operating characteristic (ROC) curves for all aneurysm variables. Volume and morphologic index of the aneurysm have the highest discriminative ability. See also Table 5. MI indicates morphologic index.

clopidogrel/prasugrel and aspirin responsiveness. Recent studies using the Multiplate Analyzer have suggested that the RISTOtest may be used to rule out vWD confidently. ${ }^{11,12}$ The test is also reportedly effective for the diagnosis and follow-up of AvWD in patients with a LVAD. ${ }^{13}$

Because both the thromboxane A2 and ADP pathways are important amplification mechanisms that greatly enhance outside-in signaling of platelets via interactions between vWF and the glycoprotein Ib-IX receptor, ${ }^{9,14}$ high-shear-stress platelet aggregation $^{15}$ and the RISTOtest ${ }^{16}$ are sensitive to aspirin and clopidogrel/prasugrel. The initial and late RISTOtest results, therefore, were below the normal limits in all our patients who underwent loading of both medications. Irreversibly suppressed thromboxane A2 (by aspirin) and ADP (by clopidogrel/prasugrel) metabolism remained stable while we took blood samples for the initial and 24-hour aggregometry tests (Table 2); thus, changes in the RISTO test during this period might be associated solely with changes in vWF activity. In the present study, the late RISTOtest results indicated reduced vWF activity in 16 patients and increased activity in 23 patients. Indeed, an increase in vWF activity in the blood is seen within 24 hours after endothelial activation (ie, injury), induced by either balloon angioplasty ${ }^{17}$ or stent implantation ${ }^{18}$ in the coronary arteries. A similar response was expected in all patients in our study population; this occurred, however, in only 23 patients with "small" aneurysms, whereas vWF activity was reduced in the remaining 16 patients with "large" aneurysms due to the predominant role of FDSs.

Because computational fluid dynamics simulations show supraphysiologic characteristics while the flow passes through stent struts across the aneurysmal neck, ${ }^{4}$ the total sheared blood volume (and number of shear-induced elongated vWF multimers) might correlate with both the aneurysmal neck area and aneurysmal volume. Therefore, we established a novel variable to predict the presence of reduced vWF activity. The morphologic index of the aneurysm is determined as the aneurysmal neck area multiplied by volume. Receiver operating characteristic curve analysis showed that reduced vWF activity was best predicted equally by both the novel morphologic index and the volume of the aneurysm (Table 5 and Fig 1 and 2). As expected, 1 fusiform aneurysm was observed in the reduced vWF group due to a relatively large neck length/area.

Adjunctive coiling is the most frequently used measure to protect aneurysms from delayed rupture in patients undergoing FDS implantation. Although adjunctive coiling does not eradicate this complication, ${ }^{19}$ we applied a preventive coiling strategy in 6 of 39 patients whose aneurysms were deemed vulnerable to rupture/ growth because of their relatively large size. The morphologic indices and volumes of these patients predicted that they would be in the reduced vWF group, and this prediction was correct (Fig 1). Our results show that adjunctive suboptimal coiling may not nullify the reduction in vWF activity after FDS implantation.

One problem that continues to be encountered after FDS implantation is late aneurysm rupture, a life-threatening complication that occurs in approximately $3 \%$ of patients. ${ }^{6}$ The accumulation of white thrombi within the aneurysmal lumen, with subsequent lysis of and/or extravasations from the aneurysmal wall due to local vasoactive and destructive factors secreted by the recruited cells, appears to be the most acceptable explanation for late aneurysm rupture after FDS implantation. ${ }^{20,21} \mathrm{MR}$ imaging findings after FDS implantation (ie, perianeurysmal edema and circumferential mural enhancement after contrast administration) further support the concept of thrombus-initiated aneurysmal wall injury. ${ }^{22}$ Moreover, approximately half of such ruptured aneurysms are larger than $2.5 \mathrm{~cm}$ in diameter, ${ }^{19}$ implying that a heavier thrombus load (ie, a larger aneurysm) may be a predisposing factor.

One other life-threatening complication that occurs after FDS implantation in approximately $3 \%$ of patients is delayed parenchymal bleeding. ${ }^{6}$ More than $80 \%$ of delayed intraparenchymal bleeding occurs within the vascular territory having that of treated aneurysms, ${ }^{19}$ supporting a deleterious role of FDS and/or its implantation procedure. In a previous study documenting MR imaging findings soon (within 2-9 days) after elective FDS implantation, ${ }^{23}$ new DWI lesions in the brain were present in 13 of 24 patients (57\%). Additionally, new susceptibility effect foci were definitively found in $8(38 \%)$ and putatively found in 6 (28\%) of 21 patients (66\% overall); these foci nearly always occurred distally within the vascular territory undergoing FDS 
treatment. On delayed MR imaging, all susceptibility effect foci remained unchanged in size, number, and morphology, confirming that the stable lesions represented microhemorrhages. Another recent strictly controlled clinical study ${ }^{24}$ identified a temporal/causative association between DWI lesions and susceptibility effect foci (microhemorrhage) after FDS implantation. Those investigators found new DWI lesions in 27 of 30 patients (90\%), whereas microhemorrhage was not detected on MR imaging at 1 day after stent placement. At 6 months, MR imaging indicated new microhemorrhagic foci in 11 of 30 patients (36\%); notably, 14 of 18 (77\%) microhemorrhagic foci had originated from previous ischemic DWI lesions, while the remaining 4 were de novo. Because de novo emergence of ipsilateral ischemic foci has been observed on MR imaging up to 1 year after FDS therapy, ${ }^{25}$ these remaining 4 microhemorrhagic foci might also have resulted from newly generated ischemic foci during the follow-up period. Taken together, a shear-induced sustained increase in vWF activity coming from a downstream stented vessel may be responsible for the initiation of a series of events leading to microthrombus formation in the ipsilateral microcirculation of the brain with subsequent hemorrhagic transformation.

Some vascular devices can create high shear in the blood, thus deteriorating strictly controlled vWF activity balance and causing clinical and laboratory evidence of type 2A AvWD. LVAD and extracorporeal membrane oxygenation are typical examples. Neuroimaging studies of patients with these types of shear-generating vascular devices may help explain the above-mentioned MR imaging findings after FDS. An MR imaging examination conducted on patients after LVAD explantation (mean duration, $2.43 \pm 1.08$ years) revealed cerebral microbleeds in $97 \%$ of patients; the number of microbleeds was positively correlated with the hemorrhagic stroke episode during LVAD support. ${ }^{26}$ Similarly, symmetric white matter microhemorrhagic foci have been reported on MR imaging, even after days or weeks of extracorporeal membrane oxygenation maintenance. ${ }^{27,28}$ These findings are consistent with previous MR imaging studies conducted on patients with FDS. ${ }^{23,24}$ We hypothesized that supraphysiologic shear-induced activation (ie, elongation) of vWF serves as an initiator of a cascade of events that eventually lead to delayed hemorrhagic complications after FDS implantation. Our result is the first to show the possible link between FDS implantation and AvWD.

Important limitations of this study are its retrospective design and limited number of patients. Another important limitation is that it was constructed on the basis of a single laboratory investigation. Lack of computational fluid dynamics analysis of the aneurysms is another limitation. Therefore, a prospective series supported by other vWF assays (both multimer activity and length) and computational fluid dynamics analysis would be useful to validate the present findings.

\section{CONCLUSIONS}

This is the first study to show that reduced vWF activity may occur after FDS implantation, especially in patients with relatively large intracranial aneurysms. A reduction in vWF activity was best predicted by the morphologic index and volume of the aneurysm. Because the reduced vWF activity accompanying the hemorrhagic disorder is the typical characteristic of vascular device-related AvWD, we hypothesize by analogy that after FDS implantation, shear-induced activation of vWF may be causally linked to the initiation of a cascade of events leading to delayed hemorrhagic complications.

Disclosures: Mustafa Parildar-UNRELATED: Payment for Lectures Including Service on Speakers Bureaus: Transarterial Radioembolization Lectures and Satellite Symposium. * *Money paid to institution.

\section{REFERENCES}

1. Horiuchi H, Doman T, Kokame K, et al. Acquired von Willebrand syndrome associated with cardiovascular diseases. I Atheroscler Thromb 2019;26:303-14 CrossRef Medline

2. Nascimbene A, Neelamegham S, Frazier OH, et al. Acquired von Willebrand syndrome associated with left ventricular assist device. Blood 2016;127:3133-41 CrossRef Medline

3. Kalbhenn J, Schlagenhauf A, Rosenfelder S, et al. Acquired von Willebrand syndrome and impaired platelet function during venovenous extracorporeal membrane oxygenation: rapid onset and fast recovery. J Heart Lung Transplant 2018;37:985-91 CrossRef Medline

4. Xiang J, Ma D, Snyder KV, et al. Increasing flow diversion for cerebral aneurysm treatment using a single flow diverter. Neurosurgery 2014;75:286-94 CrossRef Medline

5. Zhang P, Liu X, Sun A, et al. Hemodynamic insight into overlapping bare-metal stents strategy in the treatment of aortic aneurysm. J Biomech 2015;48:2041-46 CrossRef Medline

6. Zhou G, Su M, Yin YL, et al. Complications associated with the use of flow-diverting devices for cerebral aneurysms: a systematic review and meta-analysis. Neurosurg Focus 2017;42:E17 CrossRef Medline

7. Zanaty M, Chalouhi N, Tjoumakaris SI, et al. Aneurysm geometry in predicting the risk of rupture: a review of the literature. Neurol Res 2014;26:308-13 CrossRef

8. Oran I, Cinar C, Bozkaya H, et al. Tailoring platelet inhibition according to multiple electrode aggregometry decreases the rate of thrombotic complications after intracranial flow-diverting stent implantation. J Neurointerv Surg 2015;7:357-62 CrossRef Medline

9. Springer TA. Von Willebrand factor, Jedi knight of the bloodstream. Blood 2014;124:1412-25 CrossRef Medline

10. Schneider SW, Nuschele S, Wixforth A, et al. Shear-induced unfolding triggers adhesion of von Willebrand factor fibers. Proc Natl Acad Sci U S A 2007;104:7899-903 CrossRef Medline

11. Schmidt DE, Bruzelius M, Majeed A, et al. Whole blood ristocetinactivated platelet impedance aggregometry (Multiplate) for the rapid detection of von Willebrand disease. Thromb Haemost 2017;117:1528-33 CrossRef Medline

12. Nummi V, Lassila R, Joutsi-Korhonen L, et al. Comprehensive reevaluation of historical von Willebrand disease diagnosis in association with whole blood platelet aggregation and function. Int $J \mathrm{Lab}$ Hematol 2018;40:304-11 CrossRef Medline

13. Steinlechner B, Dworschak M, Birkenberg B, et al. Platelet dysfunction in outpatients with left ventricular assist devices. Ann Thorac Surg 2009;87:131-38 CrossRef Medline

14. Du X. Signaling and regulation of the platelet glycoprotein Ib-IXV complex. Curr Opin Hematol 2007;14:262-69 CrossRef Medline

15. Matsumoto M, Kawaguchi S, Ishizashi $H$, et al. Platelets treated with ticlopidine are less reactive to unusually large von Willebrand factor multimers than are those treated with aspirin under high shear stress. Pathophysiol Haemost Thromb 2005;34:35-40 CrossRef Medline

16. Annick Ankri A, Baranger A, Martin-Toutain I, et al. Impaired ristocetin-induced platelet aggregation in whole blood assessed with a Multiplate analyzer suggests a new mechanism of antiplatelet effect of aspirin and clopidogrel. Int J Lab Hem 2011;118:3362 CrossRef 
17. Hojo Y, Ikeda U, Katsuki T, et al. Release of endothelin $\mathbf{1}$ and angiotensin II induced by percutaneous transluminal coronary angioplasty. Cathet Cardiovasc Interv 2000;51:42-49 CrossRef Medline

18. Marketou M, Kochiadakis GE, Giaouzaki A, et al. Long-term serial changes in platelet activation indices following sirolimus elution and bare metal stent implantation in patients with stable coronary artery disease. Hellenic J Cardiol 2017;58:43-48 CrossRef Medline

19. Rouchaud A, Brinjikji W, Lanzino G, et al. Delayed hemorrhagic complications after flow diversion for intracranial aneurysms: a literature overview. Neuroradiology 2016;58:171177 CrossRef Medline

20. Kulcsar Z, Houdart E, Bonafe A, et al. Intra-aneurysmal thrombosis as a possible cause of delayed aneurysm rupture after flow-diversion treatment. AJNR Am J Neuroradiol 2011;32:20-25 CrossRef Medline

21. Raymond J, Darsaut TE, Kotowski M, et al. Thrombosis heralding aneurysmal rupture: An exploration of potential mechanisms in a novel giant swine aneurysm model. AJNR Am J Neuroradiol 2013;34:346-53 CrossRef Medline

22. Berge J, Tourdias T, Moreau JF, et al. Perianeurysmal brain inflammation after flow diversion treatment. AJNR Am J Neuroradiol 2011;32:1930-34 CrossRef Medline
23. McGuinness BJ, Memon S, Hope JK. Prospective study of early MRI appearances following flow-diverting stent placement for intracranial aneurysms. AJNR Am J Neuroradiol 2015;36:943-48 CrossRef Medline

24. Nakae R, Nagaishi M, Kawamura Y, et al. Microhemorrhagic transformation of ischemic lesions on $\mathrm{T} 2{ }^{*}$-weighted magnetic resonance imaging after Pipeline Embolization Device treatment. $J$ Neurosurg 2018 May 1:1-8 [Epub ahead of print] CrossRef Medline

25. Safain MG, Roguski M, Heller RS, et al. Flow diverter therapy with the Pipeline Embolization Device is associated with an elevated rate of delayed fluid-attenuated inversion recovery lesions. Stroke 2016;47:789-97 CrossRef Medline

26. Yoshioka D, Okazaki S, Toda K, et al. Prevalence of cerebral microbleeds in patients with continuous-flow left ventricular assist device. J Am Heart Assoc 2017;6:e005955 CrossRef Medline

27. Le Guennec L, Bertrand A, Laurent C, et al. Diffuse cerebral microbleeds after extracorporeal membrane oxygenation support. $A m \mathrm{~J}$ Respir Crit Care Med 2015;191:594-96 CrossRef Medline

28. Liebeskind DS, Sanossian N, Sapo ML, et al. Cerebral microbleeds after use of extracorporeal membrane oxygenation in children. $J$ Neuroimaging 2013;23:75-78 CrossRef Medline 